\title{
A Review of the Clinical Usefulness of Selective Laser Trabeculoplasty in Exfoliative Glaucoma
}

\author{
Andreas Katsanos · Anastasios G. Konstas • Dimitrios G. Mikropoulos • \\ Luciano Quaranta · Irini C. Voudouragkaki - Georgios P. Athanasopoulos • \\ Ioannis Asproudis · Miguel A. Teus
}

Received: February 21, 2018 / Published online: April 11, 2018

(C) The Author(s) 2018

\section{ABSTRACT}

In the last decade, selective laser trabeculoplasty (SLT) has been commonly used in the management of several different types of glaucoma, as either primary or adjunct therapy. The technique has an excellent safety profile and is at least as effective as argon laser trabeculoplasty. Although the actual mechanism of action of SLT remains unclear, evidence has shown that it

Enhanced content To view enhanced content for this article go to https://doi.org/10.6084/m9.figshare. 6005051 .

A. Katsanos · I. Asproudis

Department of Ophthalmology, University of Ioannina, Ioannina, Greece

A. G. Konstas $(\bowtie)$ · I. C. Voudouragkaki ·

G. P. Athanasopoulos

1st University Department of Ophthalmology, Aristotle University of Thessaloniki, Thessaloniki, Greece

e-mail: konstas@med.auth.gr

A. G. Konstas · D. G. Mikropoulos

3rd University Department of Ophthalmology,

Aristotle University of Thessaloniki, Thessaloniki,

Greece

L. Quaranta

Glaucoma Unit, University of Brescia, Brescia, Italy

M. A. Teus

Department of Ophthalmology, Hospital

Universitario "Principe de Asturias," Universidad de

Alcalá, Alcalá de Henares, Spain does not induce morphologically evident trabecular meshwork alterations. SLT's non-disruptive mode of action offers the advantage of repeatability. Exfoliation glaucoma (XFG) is a secondary open-angle glaucoma with unfavorable intraocular pressure (IOP) characteristics, which typically carries a poorer long-term prognosis than primary open-angle glaucoma. Consequently, patients with XFG often need multiple medications to achieve IOP levels that prevent disease progression. Because complicated pharmacotherapy regimens undermine the long-term tolerability and compliance of patients with XFG, options such as SLT may decrease the burden of multiple therapies and ultimately improve prognosis. In fact, SLT may be a particularly attractive option in XFG because the pigment-laden trabecular tissue of these patients enhances the absorption of laser energy and thus augments the biologic effects induced by this treatment. The current article reviews the postulated mechanisms of action of SLT, discusses practical aspects of SLT therapy, and examines selected peer-reviewed literature pertaining to the clinical usefulness of this modality in XFG patients.

Keywords: ALT; Argon laser trabeculoplasty; Exfoliation syndrome; Exfoliative glaucoma; Glaucoma; Ophthalmology; Pseudoexfoliation; Selective laser trabeculoplasty; SLT 


\section{INTRODUCTION}

Over the past decade, selective laser trabeculoplasty (SLT) has become an established and popular therapeutic option worldwide for lowering intraocular pressure (IOP) in patients with ocular hypertension or open-angle glaucoma [1-4]. Since the introduction of this novel laser procedure by Latina and Park [5] more than 2 decades ago, there have been considerable research efforts to elucidate its mechanism of action, optimal treatment settings, overall efficacy, and repeatability success. Nonetheless, the precise role and clinical usefulness of SLT in the treatment algorithm of ocular hypertension or the various forms of glaucoma remain unclear.

Exfoliative glaucoma (XFG) is the most common form of secondary open-angle glaucoma worldwide [6-8]. In XFG, the worse 24-h IOP characteristics and a substantial long-term IOP variation result in a considerably worse prognosis than that of primary open-angle glaucoma (POAG) [7, 9-11]. Moreover, XFG may exhibit augmented optic nerve vulnerability to elevated pressure, thereby exacerbating the rate of optic nerve damage and visual loss [12]. Consequently, progression in XFG without therapy is three times faster than that seen in untreated POAG [11]. When therapy commences, visual stability in XFG requires a low mean target IOP (17 $\mathrm{mmHg}$ or below) [13]. Since severe functional damage at presentation is common in XFG, it is generally challenging to obtain the desired target IOP in XFG with topical monotherapies [7]. Thus, combined medical therapy is often necessary in the treatment algorithm of XFG, and the clinician often faces the need for additional therapy options within the course of XFG management $[7,8,14]$.

Successful medical therapy balances good efficacy, tolerability, and adherence [15]. Sadly, medical therapy success in glaucoma diminishes as we move from initial monotherapies to combined therapies [14]. In the study by Barnebey and Robin [16] who monitored adherence electronically over a period of 12 months, the adherence rate documented was consistently greater with fixed combination therapy
(60\%) than with unfixed combined therapy (43\%). At the same time, electronic monitoring of adherence to combined therapy demonstrated a rate substantially below the ideal. Consequently, the insufficient long-term success with current combined medical therapy is due to the fact that it is more difficult to attain the combination of good efficacy, tolerability, and adherence with multiple therapies [14]. By reducing adherence, ocular surface health, and tolerability, combined regimens undermine the overall success of medical therapy [15]. The difficulty in controlling IOP medically in XFG explains the higher probability for filtration surgery in many XFG patients [7, 12].

To achieve greater success in long-term glaucoma care, it is essential to reduce the burden of chronic combined medical therapy (i.e., fewer drops) and diversify care by employing alternative therapy options, SLT, or minimally invasive glaucoma surgery (MIGS). This also implies that we should consider laser and specifically SLT not as a last resort, when medical therapy has failed, but as a second or third step in our management algorithm and repeat it if necessary [17-20]. Indeed, SLT may assist in delaying or even avoiding filtration surgery, which could be important for some patients [4, 21-23].

Importantly, emerging evidence suggests that SLT may obtain greater IOP reduction and possibly greater success in XFG [24-26]. As later discussed in detail, SLT may be particularly suitable for patients with XFG, as this technique depends on the absorption of laser energy by pigmented tissue of the trabecular meshwork. It is well established that XFG patients manifest a heavily pigmented meshwork and pigment accumulation constitutes a key mechanism for IOP elevation in XFG $[5,6,27]$. To date, obstruction of the trabecular meshwork by pigment and exfoliation material is considered the most likely cause of elevated IOP in XFG $[6,8]$.

This review article provides a brief overview of this novel treatment modality. We discuss the postulated mechanisms of action, practical aspects of SLT therapy, and clinical usefulness of SLT in XFG and examine selected peer-reviewed literature pertaining to the efficacy and safety of SLT in patients with XFG. This article is based 
on previously conducted studies and does not contain any studies with human participants or animals performed by any of the authors.

\section{MECHANISM OF ACTION}

Even today the precise mechanism through which SLT exerts its IOP-lowering effect remains elusive. Experimental evidence has suggested that SLT induces a number of biologic rather than mechanical effects that ultimately increase trabecular meshwork outflow facility [28]. These biologic effects involve cytokine secretion, matrix metalloproteinase induction, macrophage recruitment, increased cell division, and repopulation of burn sites [28-31].

Holló [29] compared the morphologic effects induced by argon laser trabeculoplasty (ALT) in one eye and SLT in the fellow eye of patients with bilateral failed trabeculoplasty. Transmission electron microscopy was used for the examination of six trabecular meshwork tissue blocks removed during trabeculectomy performed 3 months after laser therapy. In eyes treated with ALT, extensive histologic damage of the uveoscleral meshwork was evident at the areas of the laser spots. Although the untreated areas were characterised by intact collagen fibers, a superficial monolayer of activated, migrating endothelial cells with several microprocesses was observed in these non-lasered locations. In those eyes treated with SLT, the trabecular beams and the structure of the uveoscleral meshwork had completely disappeared at the locations of the laser spots [29]. Additionally, in regions adjacent to the lasered areas, localized shrinkage, fine scarring, phagocytosis of pigment granules, and deformed trabecular endothelial cells were observed at the transition between the uveoscleral and corneoscleral meshwork. However, no heat-damaged collagen, juxtacanalicular tissue alterations, or endothelial membrane could be seen. This could be clinically relevant as the expanding superficial endothelial layer that covers the trabecular meshwork has been considered a cause of outflow reduction and treatment failure [30]. In a more recent ex vivo study, SooHoo et al. [31] employed corneoscleral tissue samples from human cadaver eyes without glaucoma to determine the morphologic changes induced by ALT and SLT. The authors used light microscopy and transmission and scanning electron microscopy to examine the tissue sections after spots of different energy levels were delivered with ALT and SLT. Following SLT, light microscopy revealed intact trabecular beams similar to the ones observed in untreated tissue, whereas transmission electron microscopy showed disrupted trabecular meshwork cells with extracellular pigment granules. Scanning electron microscopy of sections treated with high-energy SLT $(2.0 \mathrm{~mJ})$ showed tissue destruction with scrolling of trabecular beams at the edges of the laser spots. On the other hand, sections treated with ALT showed significant tissue disruption and loss of trabecular architecture on light microscopy, loss of cell integrity and dispersion of pigment granules on transmission electron microscopy, and tissue destruction with crater formation on scanning electron microscopy [31]. Overall, the promise of SLT in reducing injury to the trabecular meshwork in patients with glaucoma (and especially XFG) is a key theoretical and experimental advantage but remains to be proven clinically.

\section{PRACTICAL ASPECTS OF SLT THERAPY}

Typically, SLT is performed with approximately 50 applications over $180^{\circ}$ or, increasingly, 100 applications over $360^{\circ}$ of the trabecular meshwork. As spot size $(400 \mu \mathrm{m})$ and duration (3 ns) are fixed, the surgeon only needs to titrate laser energy to a level when microbubbles become evident or to the energy level immediately below the threshold that produces microbubbles $[21,22]$. The energy level is initially set at approximately $0.8 \mathrm{~mJ}$ and modified depending on the tissue reaction in the range between 0.3 and $1.7 \mathrm{~mJ}$. As SLT energy is preferentially absorbed by melanin pigment, increased trabecular pigmentation has been found to enhance the efficacy of SLT $[27,32]$. Therefore, lower energy settings are advised in highly pigmented angles. It is possible to see a 
paradoxical IOP increase after SLT with standard energy levels in eyes with pigmentary glaucoma and XFG [33].

Some evidence suggested that there was no significant efficacy difference if one treats $180^{\circ}$ as opposed to $360^{\circ}$ of the trabecular meshwork in open-angle glaucoma patients [34, 35]. In contrast, a study by Shibata et al. [36] established that SLT over $360^{\circ}$ achieves lower pressures than $180^{\circ}$. Similarly, another study determined that SLT over $360^{\circ}$ of the trabecular meshwork was more likely to achieve lower pressure and reduced inter-visit IOP variation in patients with POAG or ocular hypertension [37]. Sadly, no study to date has addressed this issue specifically in patients with XFG. Although traditionally $360^{\circ}$ or $180^{\circ}$ of the trabecular meshwork are treated, SLT therapy limited to only $90^{\circ}$ of the meshwork has also been attempted [32]. The authors of this prospective study conducted on open-angle glaucoma patients (of whom approximately $50 \%$ had XFG) reported that SLT was just as effective with 25 laser spots over $90^{\circ}$ as 50 laser spots applied over $180^{\circ}$ of the trabecular meshwork [32]. This albeit limited evidence may imply that in XFG the heavily pigmented meshwork could justify "less SLT therapy" with reduced levels of energy, and the area of laser treatment may prove just as effective with fewer side effects (i.e., postoperative pressure spikes).

Although an individual patient's response to SLT is difficult to predict, the ocular hypotensive effect is generally proportionate to the level of pre-laser baseline IOP [38, 39]. In addition, the patient's response to SLT in one eye serves as a reasonably accurate predictor of the response that could be anticipated in the fellow eye [40].

One of the proposed advantages of SLT is its repeatability in eyes that have previously undergone ALT [41] or SLT [17-20, 42]. A prospective randomized trial examined the effect of treatment location on repeat SLT in eyes with XFG or POAG in which previous SLT had not achieved an IOP reduction $>20 \%$ [42]. Eyes with previous treatment of the inferior half of the trabecular meshwork were randomized to retreatment over the previously treated (i.e., inferior) part or over the untreated (i.e., superior) area of the trabecular meshwork [42]. Importantly, this study demonstrated that both SLT options were effective and, consequently, the IOP was significantly reduced in both groups. Moreover, there was no significant difference in efficacy between the two laser groups.

In a meta-analysis, Wang et al. [43] demonstrated that glaucoma patients who have undergone $360^{\circ}$ of previous laser still benefit from SLT, manifesting IOP decreases similar to those of patients who were naïve to prior laser therapy. In addition, this meta-analysis showed that SLT did not differ from ALT with respect to other important clinical outcomes, such as the number of IOP-lowering medications and the success rate. In another study, Damji et al. [44] highlighted that patients with previous failed ALT or SLT had a better outcome when treated with SLT than with ALT. However, whether SLT has better long-term efficacy than ALT in repeat laser trabeculoplasty treatments remains unclear.

Thus, it appears that re-treatment with SLT is a valuable clinical option with clinically meaningful and predictable effectiveness when performed in previously treated patients. How many times SLT can or should be repeated remains unknown. In XFG specifically, SLT retreatment could be a particularly valuable option as there is on-going accumulation of pigment upon and within the trabecular meshwork [6]. More evidence is needed in the future to elucidate when and how SLT re-treatment should be selected in XFG patients.

Following SLT therapy, glaucoma medications are usually continued since in most patients SLT is selected to further reduce the target IOP. Over time, however, when SLT is successful, the daily burden of medical therapy can be diminished with fewer medications used and therefore reduced administration of drops. There is as yet limited published evidence on the overall success of SLT in simplifying or reducing combined therapy regimens. The success of SLT regarding enhancement of patients' overall quality of life, convenience, real-life adherence, tolerability, and therapy outcome remains to be elucidated. Future studies should pay attention to clinical endpoints other than only IOP reduction. Optimizing these 
endpoints would be of greater value in XFG patients since they are generally older and often require more medications to control IOP because of worse 24-h IOP characteristics [7-10].

To date, there is no consensus on the necessity of antiinflammatory medications in the immediate post-laser period. Some surgeons prescribe a topical steroid for a few days to diminish inflammation and improve comfort. Others do not, based on the notion that the mild inflammatory reaction induced by SLT may be desirable because it facilitates tissue biomechanical effects that increase trabecular function. A randomized double-masked, placebo-controlled trial evaluated patients treated with topical prednisolone, ketorolac, or placebo q.i.d. for 5 days following SLT [45]. No significant differences in anterior chamber inflammation scores were noted among the treatment groups in the immediate postoperative period. Moreover, the authors did not observe significant differences in the IOP-lowering effect for up to 1 year after SLT treatment.

Postoperatively, IOP spikes (and even prolonged IOP elevation that eventually necessitated surgical intervention) have occasionally been reported after SLT. In a short series of four patients, IOP elevations that lasted between 4 days and 3 months were described following SLT treatment in eyes with heavy angle pigmentation [33]. Two of these patients had previously undergone ALT and another had sustained previous ocular trauma. In this small case series, trabeculectomy was eventually required for three of these SLT-treated patients. Consequently, lower power settings and close postoperative follow-up are warranted in patients with heavily pigmented angles. Another short series recently described five cases of XFG that eventually required trabeculectomy because of recalcitrant IOP elevation after SLT [46]. Interestingly, all five patients exhibited diffuse corneal edema, which in some cases persisted despite the normalization of the IOP. Two of the patients suffered severe endothelial damage and eventually required corneal transplantation. The pathomechanism of corneal edema in these cases was unclear; it may have been caused by high IOP, but it should be noted that to date the potential effect of SLT on corneal endothelial health (if any) has not been adequately determined [47].

Another matter of debate has been the possible influence of chronic prostaglandin therapy on the subsequent efficacy of SLT. Some investigators [48-50] observed no relationship between SLT outcome and previous prostaglandin use, but in at least one report the concomitant use of prostaglandins was associated with greater IOP reduction in the 1st month following SLT [51]. These are all interesting clues that will be useful leads for future research specifically in patients with XFS/XFG.

A recent study [52] suggested that the use of a gonioscopy lens may not be necessary for the application of SLT energy at the trabecular meshwork, and trans-scleral treatment using the exact laser parameters as in conventional procedures is equally effective. Nevertheless, as this preliminary study only included a small number of treated subjects (trans-scleral group: $n=14$; gonioscopy lens group: $n=14$ ), confirmation of these findings in larger studies is mandatory before this method can be applied in daily practice.

\section{EFFICACY OF SLT IN EYES WITH XFG}

\section{SLT as Primary Therapy}

Unfortunately, to date, there has not been a primary SLT study specifically in XFG. In a prospective trial, Melamed et al. [24] investigated the efficacy of SLT as a primary therapy in 45 eyes of 31 patients with newly diagnosed open-angle glaucoma or ocular hypertension. This study included 29 cases with POAG and 5 with XFG. All patients had $180^{\circ}$ of their trabecular meshwork treated with SLT. After a mean follow-up time of 11 months, the mean pre-laser IOP of the treated cohort $(25.5 \pm 2.5 \mathrm{mmHg})$ was significantly reduced by primary SLT to $17.9 \pm 2.8 \mathrm{mmHg} \quad(30 \%$ reduction; $P<0.001)$. It is worth noting that in the smaller XFG cohort the mean IOP reduction was noticeably greater (from $28.6 \pm 3.2$ to $16.8 \pm 0.8 \mathrm{mmHg} ; 41 \%$ reduction; $P=0.001$ ) than that seen in the POAG group (from 
$25.5 \pm 2.0$ to $18.5 \pm 2.8 \mathrm{mmHg} ; 27 \%$ reduction; $P<0.001)$.

Subsequently, the efficacy of primary SLT was investigated in a prospective trial that included 18 cases with XFG and 19 cases with POAG [2]. These authors also treated $180^{\circ}$ of the trabecular meshwork in all their patients. Failure was defined as the need for medical, surgical, or further laser treatment or the return of treated IOP within $3 \mathrm{mmHg}$ of the baseline pressure. The mean pre-laser IOPs in the XFG and POAG patients were $25.5 \pm 3.4$ and $23.2 \pm 3.0 \mathrm{mmHg}$, respectively. After 30-42 months of follow-up, XFG patients who were not considered failures had a mean IOP of $18.3 \pm 4.7 \mathrm{mmHg}$ (mean IOP reduction: $5.3 \mathrm{mmHg}$ ), whereas patients with POAG who were not considered failures exhibited a mean IOP of $17.6 \pm 2.8 \mathrm{mmHg}$ (mean IOP reduction: $5.7 \mathrm{mmHg}$ ). The cumulative probability of success was similar for the XFG and POAG groups at $74 \%$ and $77 \%$, respectively, at the end of follow-up. Although no major adverse events were noted, temporary IOP spikes $>5 \mathrm{mmHg}$ occurred in $28 \%$ of XFG patients and $21 \%$ of POAG patients, and one patient with XFG $(5.5 \%)$ exhibited an IOP spike $>10 \mathrm{mmHg}$.

Therefore, these two small studies did not agree on the relative efficacy of primary SLT in POAG compared with XFG. The inconsistency in these two investigations may be due to design differences and methodologic weaknesses. The study by Melamed et al. [24] included only five cases with XFG. In the study by Shazly et al. [2], the authors reported IOP results at the end of the follow-up period only for eyes that were not considered "failures," but not for the whole treatment group. It is also noteworthy that in this study the untreated baseline IOP of the XFG group was rather low $(25.5 \pm 3.4 \mathrm{mmHg})$, which is not typical of other XFG groups or other published reports $[9,24]$. More evidence from controlled, adequately powered studies is needed to adequately describe the efficacy of primary SLT in XFG.

To date, several aspects of primary SLT therapy remain poorly defined. For example, the optimal level of energy, or the potential added value of $360^{\circ}$ treatment, has not been determined. Furthermore, the 24-h efficacy of primary SLT in XFG versus untreated baseline, or compared with other commonly used therapeutic options, remains to be elucidated [23]. Of particular interest may be the impact of SLT on the 24-h IOP parameters in patients with XFG. The quality of 24-h IOP control with SLT versus medical or surgical treatment may reflect the future probability of progression in eyes with XFG $[7,9,13,53]$. Finally, since in XFG there is continuous accumulation of pigment at the trabecular meshwork, it may be critical to determine the optimal timing and likely efficacy of future SLT re-treatments.

\section{SLT as Adjunct Therapy}

To date, SLT has been extensively investigated as adjunct therapy to patients insufficiently controlled on medical therapy. In a small prospective trial that included ten XFG and ten POAG patients, Gracner [54] investigated the efficacy of $180^{\circ}$ SLT therapy. All participants were uncontrolled with maximum medical therapy. The mean pretreatment IOP was not statistically different for the two groups (23.6 vs. $22.8 \mathrm{mmHg}$ for the XFG and the POAG groups, respectively). The mean follow-up was 12.0 months for the XFG group and 13.5 months for the POAG group. The authors observed that the degree of IOP lowering up to the final visit at 18 months was smaller for the XFG group at all time points, but this difference became statistically significant at the 12-month visit $(22.0 \%$ vs. $30.6 \% ; P<0.05)$. These results should be interpreted with caution, however, because this small study was beset by high attrition (12-month visit: only five XFG cases and eight POAG cases; 18 -month visit: only four XFG cases vs. four POAG cases). These results contrast evidence from a subsequent study, which compared 17 POAG/NTG patients with 11 XFG patients. All subjects were poorly controlled on medical therapy and were followed up for 2 years [25]. These authors documented that at the end of the follow-up period, XFG patients achieved a significantly greater $28.2 \%$ IOP reduction from baseline (from a mean IOP of $18.8 \mathrm{mmHg}$ ) with a mean 1.88 topical medications compared with $15.2 \%$ reduction from 
the pre-laser value $(17.1 \mathrm{mmHg})$ with a mean 1.27 topical medications for patients with POAG or NTG.

Subsequently, Goldenfeld et al. [55] investigated the efficacy and safety of SLT as an adjunct therapy option specifically in XFG patients. This 12 -month trial included 57 eyes of 57 patients uncontrolled on maximal medical therapy. Patients were treated over $180^{\circ}$ of the trabecular meshwork. Following SLT, the mean IOP decreased from $26.01 \pm 2.5$ to $17.8 \pm 2.8 \mathrm{mmHg} \quad(31.5 \% \quad$ reduction; $P<0.001)$, whereas the mean number of medications necessary for IOP control was reduced from 2.8 to 2.3 at 12 months. At the end of the follow-up period, $90 \%$ of XFG patients had a mean IOP lower than $21 \mathrm{mmHg}$ and $66 \%$ of XFG patients achieved a mean IOP lower than $18 \mathrm{mmHg}$ with or without medication. Five eyes $(8.8 \%)$ required trabeculectomy and were therefore considered failures. Two eyes (3.5\%) with heavy trabecular pigmentation had an IOP spike $\geq 5 \mathrm{mmHg}$ within $1 \mathrm{~h}$ following SLT. In both cases, the IOP spikes resolved with medical therapy within 2 days.

In a prospective comparative trial [56], Ayala and Chen investigated the efficacy of $90^{\circ}$ adjunct SLT treatment in patients with XFG $(n=30)$ compared with POAG $(n=30)$. The authors also determined the inflammation induced by the treatment using a flare meter. Forty-five patients were treated with SLT for the first time, eight were treated for the second time, and seven were treated for the third time. All patients continued their pre-laser antiglaucoma medication and did not receive any antiinflammatory or IOP-lowering treatment after the procedure. The mean pre-laser IOP for the XFG group was $24.8 \pm 0.0 \mathrm{mmHg}$ with a mean 2.04 medications, whereas the mean pre-laser IOP for the POAG group was $23.5 \pm 4.6 \mathrm{mmHg}$ with a mean 1.78 medications (all $P>0.05$ ). After 1 month, the IOP reduction in the XFG and the POAG groups was 6.19 and $6.87 \mathrm{mmHg}$, respectively $(P=0.27)$, and there was no significant difference in anterior chamber flare $(P=0.36)$. The authors noted that because the participants of this study had advanced glaucoma (mean defect for XFG and POAG groups: -16.1 vs. $-18.1 \mathrm{~dB}$, respectively; $P>0.05$ ), their results may not be applicable to patients with less severe damage.

The efficacy of SLT as adjunct treatment was investigated in a prospective study that enrolled 136 Iranian patients with open-angle glaucoma poorly controlled with maximal medical treatment [49]. The sample included eyes with POAG $(n=91)$, XFG $(n=22)$ and pigmentary glaucoma $(n=23)$. In this study, $360^{\circ}$ of the trabecular meshwork was lasered, and the patients received fluorometholone $0.1 \%$ b.i.d. for 3 days postoperatively. The mean follow-up was $16.6 \pm 4.3$ months. Success was defined as IOP reduction $>20 \%$ or discontinuation of at least one antiglaucoma medication due to lower IOP levels as a result of treatment. The mean preoperative IOP for the groups with POAG, XFG, and pigmentary glaucoma was $21.9 \pm 3.3$, $22.3 \pm 3.9$, and $22.1 \pm 4.9 \mathrm{mmHg}$, respectively $(P=0.9)$. At the last visit, the mean IOP in the groups with POAG, XFG, and pigmentary glaucoma was $18.3 \pm 3.8, \quad 18.3 \pm 2.9, \quad$ and $18.7 \pm 4.3 \mathrm{mmHg}$, respectively. In each group, the post-laser IOP values were significantly lower than the respective pre-laser values (all $P<0.001$ ). The mean percentage IOP reduction for the POAG, XFG, and pigmentary glaucoma groups was similar $(16.7 \%, 16.6 \%$, and $14.5 \%$, respectively; $P=0.696)$. The reduction in the number of medications was also not different among the groups $(P=0.644)$. There was no statistically significant difference in the proportion of patients satisfying the criterion of success in the three groups $(P=0.597)$. However, during follow-up, the need for repeat SLT or trabeculectomy was significantly different between glaucoma subgroups: $16.5 \%$ in eyes with POAG, $13.6 \%$ in eyes with XFG, and $26.1 \%$ in eyes with pigmentary glaucoma $(P<0.001)$. IOP spikes $>6 \mathrm{mmHg}$ were seen in six eyes ( 5 of which had pigmentary glaucoma).

In a retrospective case series, Kara et al. [26] compared the efficacy of adjunct SLT in poorly controlled patients with POAG or XFG. The authors included 48 eyes of 48 POAG patients and 37 eyes of 37 XFG patients who received $180^{\circ}$ treatment and were followed for at least 12 months. Postoperatively, all patients received one drop of brimonidine $0.2 \%$ immediately after the laser treatment and 
fluorometholone drops q.i.d for 1 week. The mean pretreatment IOP in the POAG and XFG groups was $22.7 \pm 2.1$ and $22.4 \pm 2.9 \mathrm{mmHg}$, respectively $(P=0.66)$, and the mean number of medications was $1.9 \pm 1.0$ and $2.1 \pm 1.1$, respectively $(P=0.23)$. After 12 months of follow-up, SLT significantly reduced the IOP from baseline for both glaucomas (in POAG to $18.2 \pm 1.7$ and in XFG to $16.3 \pm 3.5 \mathrm{mmHg}$; $P<0.001$ for both comparisons). However, when the two glaucoma groups were directly compared, IOP lowering was significantly greater in XFG $(-6.1 \pm 3.6$ vs. $-4.4 \pm 2.1 \mathrm{mmHg} ; P=0.002)$. In addition, at the end of follow-up, XFG patients were using significantly fewer antiglaucoma medications compared with POAG patients $(2.0 \pm 1.02$ vs. $2.4 \pm 1.30 ; P=0.036)$. These authors concluded that SLT was significantly more efficacious in XFG than POAG. Adverse events were statistically similar in both groups: IOP spikes $>5 \mathrm{mmHg}$ (POAG: 14.5\%, XFG: 27\%; $P=0.17$ ), iritis (POAG: $10.4 \%$, XFG: $24.3 \% ; P=0.13$ ), and ocular discomfort (POAG: 33.3\%, XFG: 27\%; $P=0.63)$.

A 6-month, multicenter, randomized trial evaluated the efficacy of SLT versus ALT in 76 eyes of 60 patients with exfoliation [57]. All participants were poorly controlled on maximal medical treatment according to the physician's judgment and had at least one recorded IOP reading $>24 \mathrm{mmHg}$. Previous laser trabeculoplasty was an exclusion criterion. Treatment was performed over $180^{\circ}$ of the trabecular meshwork with SLT in 45 eyes and ALT in 31 eyes. A topical non-steroidal antiinflammatory medication was administered postoperatively for 5 days. The baseline IOPs for the SLT and ALT groups were 23.1 and $25.2 \mathrm{mmHg}$, respectively $(P=0.03)$. After 6 months $(n=63)$, the reduction from baseline was 6.9 and $7.0 \mathrm{mmHg}$ for the SLT and ALT groups, respectively $(P=0.56)$, and the same proportion of patients $(73 \%)$ in both groups had achieved at least 20\% IOP reduction from baseline. Although the authors do not report the mean number of IOPlowering medications used before or after laser therapy, they mention that the change from baseline was similar for the SLT and ALT groups $(P=0.59)$ after 6 months.
In a retrospective study, Lindegger et al. [58] compared the long-term efficacy of $360^{\circ}$ SLT as adjunct therapy in eyes with XFG $(n=94)$ versus a control group with POAG or ocular hypertension $(n=250)$. The analysis included eyes that were followed up at 3-month intervals after the 3rd post-laser month for a maximum of 60 months. The baseline IOP was significantly higher in the XFG group (21.9 vs. $19.6 \mathrm{mmHg} ; P<0.01)$. There was no statistically significant difference in IOP reduction between the two groups, with the exception of the 12-month time point (XFG: $4.83 \mathrm{mmHg}$; control group: $2.68 \mathrm{mmHg} P=0.01$ ).

In a recent prospective study, Miraftabi et al. [59] compared the efficacy of adjunct SLT in 20 cases with XFG versus 28 cases with POAG. The baseline IOP was 25.7 and $22.9 \mathrm{mmHg}$ in the XFG and POAG groups, respectively $(P=0.025)$. After a single session of $360^{\circ}$ SLT treatment, the IOP of the XFG decreased to 17.9 and $21 \mathrm{mmHg}$ at the 6- and 12-month visit, respectively $(P<0.001$ and $P=0.47$, respectively). In the POAG group, the IOP decreased to 18.4 and $18.0 \mathrm{mmHg}$ at the 6- and 12-month visit, respectively $(P<0.001$ and $P=0.02$, respectively). Success (defined as IOP reduction $\geq 20 \%$ from baseline without additional medication) at the 6-month visit was achieved in a higher proportion of XFG patients $(94.1 \%$ in XFG vs. $75 \%$ in POAG, $P=0.08$ ), but at the 12 -month visit there was no difference between the two glaucomas $(25 \%$ in XFG vs. $29.1 \%$ in POAG, $P=0.9$ ).

There is some evidence to suggest that besides mean IOP, 24-h IOP characteristics such as peak pressure and fluctuation may be important predictors of glaucoma progression $[13,53,60,61]$. This may be particularly relevant in eyes with XFG because of the worse IOP characteristics in this glaucoma [7, 9]. Unfortunately, the efficacy of SLT in reducing diurnal or 24-h peak IOP and fluctuation specifically in eyes with exfoliation has not been reported to date in peer-reviewed publications. However, in eyes with ocular hypertension $(n=87)$ or openangle glaucoma ( $n=82$, of which 76 were POAG) [62], SLT was effective in reducing diurnal (8 a.m.-6 p.m.) IOP fluctuation, but this reduction of IOP fluctuation was smaller than 
the one obtained with latanoprost (41\% for SLT vs. $64 \%$ for latanoprost). Further, it is worth noting that in a 24-h study that included 26 POAG cases, Kóthy et al. [63] found that SLT significantly lowered the amplitude of $24-\mathrm{h}$ fluctuation despite the fact that none of the eyes achieved mean IOP reduction $>20 \%$.

Future 24-h studies are needed to determine how the 24-h efficacy of SLT in eyes with XFS or XFG compares with that of other treatments and in patients with POAG [23, 64] especially over the long term [65]. Determining the longterm 24-h efficacy with primary or adjunct SLT is of clinical importance when considering the possibility that SLT may provide a more favorable IOP response in eyes with XFG compared with POAG $[2,58,59]$.

\section{CONCLUSION}

By virtue of its favorable safety profile and potential for repeatability, SLT offers an attractive therapeutic choice in eyes with XFG or with XFS and ocular hypertension. To date, it remains unclear whether the theoretical advantages of SLT in XFG can be translated into actual clinical benefits. Thus, the optimal role of this laser procedure in the treatment algorithm of XFG remains to be determined. A few published clinical trials have compared the efficacy and safety of SLT in XFG versus POAG. These studies, however, had small sample sizes and returned inconclusive results. More controlled evidence is necessary to determine whether SLT is more successful in XFG and how its therapy parameters should be modified to optimize long-term outcome in XFG. In view of the biologic processes underlying its mode of action, selecting SLT at an earlier stage of the disorder would offer the best prospects for effective IOP control and better long-term therapy outcome.

\section{ACKNOWLEDGEMENTS}

Funding. No funding or sponsorship was received for this study or publication of this article.
Authorship. All named authors meet the International Committee of Medical Journal Editors (ICMJE) criteria for authorship for this article, take responsibility for the integrity of the work as a whole, and have given their approval for this version to be published.

Disclosures. Andreas Katsanos, Anastasios Georgios Konstas, Dimitrios G Mikropoulos, Luciano Quaranta, Irini C Voudouragkaki, Georgios P Athanasopoulos, Ioannis Asproudis and Miguel A Teus have nothing to disclose.

Compliance with Ethics Guidelines. This article is based on previously conducted studies and does not contain any studies with human participants or animals performed by any of the authors.

Data Availability. Data sharing is not applicable to this article as no data sets were generated or analyzed for it.

Open Access. This article is distributed under the terms of the Creative Commons Attribution-NonCommercial 4.0 International License (http://creativecommons.org/licenses/ by-nc/4.0/), which permits any noncommercial use, distribution, and reproduction in any medium, provided you give appropriate credit to the original author(s) and the source, provide a link to the Creative Commons license, and indicate if changes were made.

\section{REFERENCES}

1. Weinand FS, Althen F. Long-term clinical results of selective laser trabeculoplasty in the treatment of primary open angle glaucoma. Eur J Ophthalmol. 2006;16:100-4.

2. Shazly TA, Smith J, Latina MA. Long-term safety and efficacy of selective laser trabeculoplasty as primary therapy for the treatment of pseudoexfoliation glaucoma compared with primary open-angle glaucoma. Clin Ophthalmol. 2010;5:5-10.

3. Li X, Wang W, Zhang X. Meta-analysis of selective laser trabeculoplasty versus topical medication in the treatment of open-angle glaucoma. BMC Ophthalmol. 2015;15:107. 
4. Wong MOM, Lee JWY, Choy BNK, Chan JCH, Lai JSM. Systematic review and meta-analysis on the efficacy of selective laser trabeculoplasty in openangle glaucoma. Surv Ophthalmol. 2015;60:36-50.

5. Latina MA, Park C. Selective targeting of trabecular meshwork cells: in vitro studies of pulsed and CW laser interactions. Exp Eye Res. 1995;60:359-71.

6. Ritch R, Schlötzer-Schrehardt U. Exfoliation syndrome. Surv Ophthalmol. 2001;45:265-315.

7. Holló G, Katsanos A, Konstas AG. Management of exfoliative glaucoma: challenges and solutions. Clin Ophthalmol. 2015;9:907-19.

8. Holló G, Katsanos A. Exfoliation syndrome and exfoliative glaucoma: from genetics to surgery. In: Berhardt L, editor. Advances in Medicine and biology. New York: Nova Science Publishers Inc; 2016. p. 199-225.

9. Konstas AG, Mantziris DA, Stewart WC. Diurnal intraocular pressure in untreated exfoliation and primary open-angle glaucoma. Arch Ophthalmol. 1997;115:182-5.

10. Konstas AG, Mantziris DA, Cate EA, Stewart WC. Effect of timolol on the diurnal intraocular pressure in exfoliation and primary open-angle glaucoma. Arch Ophthalmol. 1997;115:975-9.

11. Heijl A, Bengtsson B, Hyman L, Leske MC, Early Manifest Glaucoma Trial Group. Natural history of open-angle glaucoma. Ophthalmology. 2009;116:2271-6.

12. Kim S, Sung KR, Lee JR, Lee KS. Evaluation of lamina cribrosa in pseudoexfoliation syndrome using spectral-domain optical coherence tomography enhanced depth imaging. Ophthalmology. 2013;120:1798-803.

13. Konstas AGP, Holló G, Astakhov YS, et al. Factors associated with long-term progression or stability in exfoliation glaucoma. Arch Ophthalmol. 2004;122:29-33.

14. Konstas A, Quaranta L, Katsanos A, Voudouragkaki I, Dutton G. Fixed combination therapies in glaucoma. In: Shaarawy T, Sherwood M, Hitchings R, Crowston J, editors. Glaucoma. 2nd ed. London: Elsevier; 2015. p. 583-92.

15. Holló G, Katsanos A, Boboridis KG, Irkec M, Konstas AGP. Preservative-free prostaglandin analogs and prostaglandin/timolol fixed combinations in the treatment of glaucoma: efficacy, safety and potential advantages. Drugs. 2018;78:39-64.

16. Barnebey HS, Robin AL. Adherence to fixed-combination versus unfixed travoprost $0.004 \% /$ timolol
$0.5 \%$ for glaucoma or ocular hypertension: a randomized trial. Am J Ophthalmol. 2017;176:61-9.

17. Avery N, Ang GS, Nicholas S, Wells A. Repeatability of primary selective laser trabeculoplasty in patients with primary open-angle glaucoma. Int Ophthalmol. 2013;33:501-6.

18. Polat J, Grantham L, Mitchell K, Realini T. Repeatability of selective laser trabeculoplasty. Br J Ophthalmol. 2016;100:1437-41.

19. Francis BA, Loewen N, Hong B, et al. Repeatability of selective laser trabeculoplasty for open-angle glaucoma. BMC Ophthalmol. 2016;16:128.

20. Hong BK, Winer JC, Martone JF, Wand M, Altman B, Shields B. Repeat selective laser trabeculoplasty. J Glaucoma. 2009;18:180-3.

21. Kennedy JB, SooHoo JR, Kahook MY, Seibold LK. Selective laser trabeculoplasty: an update. Asia Pac J Ophthalmol. 2016;5:63-9.

22. Leahy KE, White AJ. Selective laser trabeculoplasty: current perspectives. Clin Ophthalmol. 2015;9:833-41.

23. Konstas AGP, Quaranta L, Bozkurt B, et al. 24-h efficacy of glaucoma treatment options. Adv Ther. 2016;33:481-517.

24. Melamed S, Ben Simon GJ, Levkovitch-Verbin H. Selective laser trabeculoplasty as primary treatment for open-angle glaucoma: a prospective, nonrandomized pilot study. Arch Ophthalmol. 2003;121:957-60.

25. Zaninetti M, Ravinet E. Two-year outcomes of selective laser trabeculoplasty in open-angle glaucoma and ocular hypertension. J Fr Ophtalmol. 2008;31:981-6.

26. Kara N, Altan C, Yuksel K, Tetikoglu M. Comparison of the efficacy and safety of selective laser trabeculoplasty in cases with primary open-angle glaucoma and pseudoexfoliative glaucoma. Kaohsiung J Med Sci. 2013;29:500-4.

27. Wasyluk JT, Piekarniak-Woźniak A, Grabska-Liberek I. The hypotensive effect of selective laser trabeculoplasty depending on iridocorneal angle pigmentation in primary open angle glaucoma patients. Arch Med Sci AMS. 2014;10:306-8.

28. Kagan DB, Gorfinkel NS, Hutnik CML. Mechanisms of selective laser trabeculoplasty: a review. Clin Exp Ophthalmol. 2014;42:675-81.

29. Holló G. Argon and low energy, pulsed Nd:YAG laser trabeculoplasty. A prospective, comparative 
clinical and morphological study. Acta Ophthalmol Scand. 1996;74:126-31.

30. Alexander RA, Grierson I. Morphological effects of argon laser trabeculoplasty upon the glaucomatous human meshwork. Eye. 1989;3(Pt 6):719-26.

31. SooHoo JR, Seibold LK, Ammar DA, Kahook MY. Ultrastructural changes in human trabecular meshwork tissue after laser trabeculoplasty. J Ophthalmol. 2015;2015:476138.

32. Chen E, Golchin S, Blomdahl S. A comparison between 90 degrees and 180 degrees selective laser trabeculoplasty. J Glaucoma. 2004;13:62-5.

33. Harasymowycz PJ, Papamatheakis DG, Latina M, De Leon M, Lesk MR, Damji KF. Selective laser trabeculoplasty (SLT) complicated by intraocular pressure elevation in eyes with heavily pigmented trabecular meshworks. Am J Ophthalmol. 2005;139:1110-3.

34. Nagar M, Ogunyomade A, O'Brart DPS, Howes F, Marshall J. A randomised, prospective study comparing selective laser trabeculoplasty with latanoprost for the control of intraocular pressure in ocular hypertension and open angle glaucoma. Br J Ophthalmol. 2005;89:1413-7.

35. McAlinden C. Selective laser trabeculoplasty (SLT) vs other treatment modalities for glaucoma: systematic review. Eye. 2014;28:249-58.

36. Shibata M, Sugiyama T, Ishida O, et al. Clinical results of selective laser trabeculoplasty in openangle glaucoma in Japanese eyes: comparison of 180 degree with 360 degree SLT. J Glaucoma. 2012;21:17-21.

37. Prasad N, Murthy S, Dagianis JJ, Latina MA. A comparison of the intervisit intraocular pressure fluctuation after 180 and 360 degrees of selective laser trabeculoplasty (SLT) as a primary therapy in primary open angle glaucoma and ocular hypertension. J Glaucoma. 2009;18:157-60.

38. Hodge WG, Damji KF, Rock W, Buhrmann R, Bovell AM, Pan Y. Baseline IOP predicts selective laser trabeculoplasty success at 1 year post-treatment: results from a randomised clinical trial. Br J Ophthalmol. 2005;89:1157-60.

39. Martow E, Hutnik CML, Mao A. SLT and adjunctive medical therapy: a prediction rule analysis. J Glaucoma. 2011;20:266-70.

40. Lee JWY, Wong MOM, Wong RLM, Lai JSM. Correlation of intraocular pressure between both eyes after bilateral selective laser trabeculoplasty in open-angle glaucoma. J Glaucoma. 2016;25:e248-52.
41. Latina MA, Sibayan SA, Shin DH, Noecker RJ, Marcellino G. Q-switched 532-nm Nd:YAG laser trabeculoplasty (selective laser trabeculoplasty): a multicenter, pilot, clinical study. Ophthalmology. 1998;105:2082-8 (discussion 2089-2090).

42. Ayala M. Intraocular pressure reduction after initial failure of selective laser trabeculoplasty (SLT). Graefes Arch Clin Exp Ophthalmol. 2014;252:315-20.

43. Wang W, He M, Zhou M, Zhang X. Selective laser trabeculoplasty versus argon laser trabeculoplasty in patients with open-angle glaucoma: a systematic review and meta-analysis. PLoS One. 2013;8:e84270.

44. Damji KF, Bovell AM, Hodge WG, et al. Selective laser trabeculoplasty versus argon laser trabeculoplasty: results from a 1-year randomised clinical trial. Br J Ophthalmol. 2006;90:1490-4.

45. Jinapriya D, D'Souza M, Hollands H, et al. Anti-inflammatory therapy after selective laser trabeculoplasty: a randomized, double-masked, placebocontrolled clinical trial. Ophthalmology. 2014;121:2356-61.

46. Bettis DI, Whitehead JJ, Farhi P, Zabriskie NA. Intraocular pressure spike and corneal decompensation following selective laser trabeculoplasty in patients with exfoliation glaucoma. J Glaucoma. 2016;25:e433-7.

47. Ong K, Ong L, Ong LB. Corneal endothelial abnormalities after selective laser trabeculoplasty (SLT). J Glaucoma. 2015;24:286-90.

48. Ayala M, Chen E. The influence of topical prostaglandin analogues in inflammation after selective laser trabeculoplasty treatment. J Ocul Pharmacol Ther. 2012;28:118-22.

49. Koucheki B, Hashemi H. Selective laser trabeculoplasty in the treatment of open-angle glaucoma. J Glaucoma. 2012;21:65-70.

50. Singh D, Coote MA, O'Hare F, et al. Topical prostaglandin analogues do not affect selective laser trabeculoplasty outcomes. Eye. 2009;23:2194-9.

51. Scherer WJ. Effect of topical prostaglandin analog use on outcome following selective laser trabeculoplasty. J Ocul Pharmacol Ther. 2007;23:503-12.

52. Geffen N, Ofir S, Belkin A, et al. Transscleral selective laser trabeculoplasty without a gonioscopy lens. J Glaucoma. 2017;26:201-7.

53. Konstas AGP, Quaranta L, Mikropoulos DG, et al. Peak intraocular pressure and glaucomatous 
progression in primary open-angle glaucoma. J Ocul Pharmacol Ther. 2012;28:26-32.

54. Gracner T. Intraocular pressure response of capsular glaucoma and primary open-angle glaucoma to selective Nd:YAG laser trabeculoplasty: a prospective, comparative clinical trial. Eur J Ophthalmol. 2002; 12:287-92.

55. Goldenfeld M, Geyer O, Segev E, Kaplan-Messas A, Melamed S. Selective laser trabeculoplasty in uncontrolled pseudoexfoliation glaucoma. Ophthalmic Surg Lasers Imaging. 2011;42:390-3.

56. Ayala M, Chen E. Comparison of selective laser trabeculoplasty (SLT) in primary open angle glaucoma and pseudoexfoliation glaucoma. Clin Ophthalmol. 2011;5:1469-73.

57. Kent SS, Hutnik CML, Birt CM, et al. A randomized clinical trial of selective laser trabeculoplasty versus argon laser trabeculoplasty in patients with pseudoexfoliation. J Glaucoma. 2015;24:344-7.

58. Lindegger DJ, Funk J, Jaggi GP. Long-term effect of selective laser trabeculoplasty on intraocular pressure in pseudoexfoliation glaucoma. Klin Monatsbl Augenheilkd. 2015;232:405-8.

59. Miraftabi A, Nilforushan N, Nassiri N, Nouri-Mahdavi K. Selective laser trabeculoplasty in patients with pseudoexfoliative glaucoma vs primary open angle glaucoma: a one-year comparative study. Int J Ophthalmol. 2016;9:406-10.

60. Asrani S, Zeimer R, Wilensky J, Gieser D, Vitale S, Lindenmuth K. Large diurnal fluctuations in intraocular pressure are an independent risk factor in patients with glaucoma. J Glaucoma. 2000;9:134-42.

61. Quaranta L, Katsanos A, Russo A, Riva I. 24-hour intraocular pressure and ocular perfusion pressure in glaucoma. Surv Ophthalmol. 2013;58:26-41.

62. Nagar M, Luhishi E, Shah N. Intraocular pressure control and fluctuation: the effect of treatment with selective laser trabeculoplasty. Br J Ophthalmol. 2009;93:497-501.

63. Kóthy P, Tóth M, Holló G. Influence of selective laser trabeculoplasty on 24-hour diurnal intraocular pressure fluctuation in primary open-angle glaucoma: a pilot study. Ophthalmic Surg Lasers Imaging. 2010;41:342-7.

64. Konstas AGP, Katsanos A, Quaranta L, Mikropoulos DG, Tranos PG, Teus MA. Twenty-four hour efficacy of glaucoma medications. Prog Brain Res. 2015;221:297-318.

65. Riva I, Katsanos A, Floriani I, et al. Long-term 24-hour intraocular pressure control with travoprost monotherapy in patients with primary openangle glaucoma. J Glaucoma. 2014;23:535-40. 